\title{
Discussion of QOL (with Concise Summary of Newer Clinical Research) in Colorectal Cancer Patients Treated in a Retrospective Trial on the Effect of Surgical Resection Margins on Survival, with Relation to Operation Type
}

Nuhi Arslani ( $\square$ arslani.nuhi@gmail.com )

UKC Maribor: Univerzitetni Klinicni Center Maribor https://orcid.org/0000-0001-7543-2498

Philip R. RACHIMIS

Faculty of Medicine University of Maribor in Slovenia: Univerza v Mariboru Medicinska fakulteta

Research article

Keywords: Colorectal cancer, Distal margin, Circumferential margin, Low anterior resection, Abdominoperineal resection, Patient survival

Posted Date: October 12th, 2020

DOI: https://doi.org/10.21203/rs.3.rs-88840/v1

License: (1) This work is licensed under a Creative Commons Attribution 4.0 International License. Read Full License 


\section{Abstract}

Background: the survival of patients treated for CRC is showing a trend of increase and with it increasing concern for the QOL of the operated patient. The aim of this study was to examine the relative influence of distal and circumferential resection margin lenght on survival among a select group patients (undergoing routine treatment) in our institution, with no metastases, and not operated on as an emergency. We also hoped to provide a concise reflection of newer clinical research on the topic.

Methods: records of 303 patients, that were operated on between January 2009 and January 2014, were retrospectively reviewed for this study. Patients who underwent emergency surgery and those with metastases were excluded, which left 128 patients suitable for statistical analysis.

Results: the number of survivors was noticeably higher than the number of deaths in all groups. Analysis with the Mann-Whitney-Wilcoxon test showed statistically significant differences $(p<0.05)$ in survival between the group with different CRM-s $(p=0.01396)$, and could not be found between different DRM groups ( $p=0.1132$ ). The logistic regression model of survival analysis showed that the size CRM is an independent predictor $(p=0.0202$, AIC $=92.181)$ and the size of the DRM is not $(p=0.119$, AIC $=109.89)$.

Conclusions: a distal resection margin smaller than $1 \mathrm{~cm}$ may be oncologically acceptable in a complete R0 excision, which could theoretically help surgeons perform SSR operations with better oncological and functional results.

\section{Background}

Colorectal cancer (CRC) is currently the third most common type of neoplasm in men and fourth most common in women (cumulatively, it is the fourth most incident cancer in the world), according to GLOBOCAN 2018 data $(1,2)$. The year 2018 itself has seen about 1.8 million new cases alone. Despite that, its incidence is currently falling (at least in developed nations), and 5 year survival is showing a trend of increase (about $60 \%$ in developed countries - comparable to statistics for our cuntry and institution) $(1,3)$. This developement is due to progress in treatment, diagnosis, and adoption of national screening programs. As such, the impact of treatment on a patients quality of life $(\mathrm{QOL})$ is coming under scrutiny. Thus, the continous evolution of techniques and technologies remains important, for improvements in both oncological outcomes and QOL (4).

Most common standard resection techniques, are abdominoperineal resection (APR) and low anterior resection (LAR). The first is more invasive, while the other is potentially sphincter sparing (SSR). The main aims of modern CRC surgery is to establish a sufficient distal and circumferential resection margin (DRM and CRM), as an incomplete resection (any positive resection margin) is the main risk factor for a lethal local reccurance (5-7). The current gold standard is the principle of total mesorectal excision (TME), which aims to reduce lymphatic spread (introduced by Heald and originated from the observations of Moynihan) $(8,9)$. This is done by removing the rectal tumour with the pararectal lymph nodes. This neccesitates surgery in the areolar plane between the visceral fascia enveloping the rectum and 
mesorectum $(9,10)$. This is done trough a APR or LAR (en-block removal of the entire mesorectum). APR is indicated in cases where the tumour lies very distally (can be combined with pelvic evisceration). TME generally allows the preservation of the autonomic nervous functioning of the region - reduces the chance of post op. dysfunctions (impotence, retrograde ejaculation and urinary incontinence). TME dissections have been associated with an increased rate of anastomotic leak (11).

The negative distal resection margin (DRM) is broadly defined as a distance from the distal border of the tumour (12). As such, its effect on local reccurence and survival is still somewhat controversial, with contradicting studies $(9,12,13)$. Newer litarature contends, that even a safety margin of less than $1 \mathrm{~cm}$ is enough to ensure sufficient radicality, especially in TNM stage 1 and stage 2 lesions - which are commonly intercepted by screening programs. It is suggested that the DRM impacts survival more, than the rate of reccurance (14).This, could offer greater freedom to plan the surgery and would be especially important in cases of LAR, where a margin of only $1 \mathrm{~cm}$ is commonly required. APR is performed usually when achieving sufficient DRM is challenging. This results in a permanent stoma, which may decrease QOL (15).

The circumferential resection margin (CRM) is widely accepted to be the most important factor for local recurrence (gained prominence after the report of P. Quirke) $(4,5)$. Currently a less than $2 \mathrm{~mm}$ boundary between the tumour and mesothelial fascia is considered positive, and is associated with a high increase in local recurrence $(5,10)$. In other words, it can be defined as a tumour extension, or presence of positive lymph nodes less than $1 \mathrm{~mm}$ from the radial, non-peritonealised soft tissue border (this also increases the risk of distant metastases). It can be said that, the completeness of the TME and the quality of surgical tumour removal may be expressed as pCRM (10). However, an intact and complete pCRM may not impact rates of distant metastases (16).

The aim of this retrospective study was to examine the relative influence of resection margin lenght on the survival of a cohort of patients undergoing routine treatment in our institution (assumed to be representative of the majority patient population, after the introduction of a national screening programme), which did not have metastases, and were not operated on as an emergency. We also hope to provide a concise reflection of newer clinical research on the topic (especially QOL and oncological outcome, with reflection on the most commonly used surgical techniques), which could, in theory, contribute to the well being of our patients.

\section{Methods}

Ethical approval for this study was obtained from the equivalent of the Institutional Review board of our respective institution.

Records of 303 patients were retrospectively reviewed for this study - informed consent to use the anonymized data that is collected by the Slovenian Cancer registry as standard (survival, and tumour characteristics on samples sent to pathology), was obtained before the treatment itself (none of the patients refused participation). Patients in the cohort were born between 1925 and 1971 (on avarage 
61 years old) and were operated on between January 2009 and January 2014, due to colorectal carcinoma. Those who underwent surgery, as an emergency, and those with metastases were excluded from the cohort (175 patients in total), which left 128 patients suitable for statistical analysis. The patients in were processed and later treated in accordance with the standard ESMO guidelines for colorectal cancer - due to cohort characteristics, they were all operated on with a procedure, that can be classed as either a standard APR, or LAR.

The exclusions to the patient cohort were deemed necessary, as late TNM stage cancers, may confound data - neccesitatting multivisceral surgery (17), so a R0 resection can rarely be performed, additionaly late stage tumours have their own independant variables $(18,19)$. In hindsight, the decision also made sense, due to the lower incidence of high stage cancers, because of earlier diagnostics (national screening program - SVIT), which would further reduce sample sizes into irrelevance.

The bowel margins were measured in specimens that were pinned (with care, not to stretch the sample) to a board and fixed with formaline for microscopy. The donut cutting edges were not included in those measurements.

Survival data on patient survival was retrieved from the Cancer Registry of the Republic of Slovenia. It was analyzed on R-3.4.4 for windows (32/64 bit) - CRAN 16 was used for data analysis. The data was first keyed in MS Excel @ before converting it into R-3.4.4 for analysis.

The data was based on 5-year of survival - patients who survived the entirety of 5 years, were counted in the live group, those who died before that, were counted in the dead group - thus the e.g. Kaplan-Meier method or Cox regression for survival analysis, could not be used to analyze the standard 'time-to-event' data. Rather the Mann-Whitney-Wilcoxon test was used to determine statistically significant differences in the survival of groups of patients with a circumferential margin $\leq 2 \mathrm{~mm}$ and $>2 \mathrm{~mm}$ and groups of patients with a distal margin $\leq 1 \mathrm{~cm}$ and $>1 \mathrm{~cm}$. Logistic regression was used to determine the best model corresponding to the mortality-to- circumferential or distal margin ratio.

\section{Results}

The study analyzed the CRM and DRM of 128 patients (43 women and 85 men) who underwent surgery using the »Dixon« method (LAR - 94 patients) or »Miles« (APR - 34 patients). Patients were divided into groups with a CRM of $\leq 2 \mathrm{~mm}$ (17 patients) and $>2 \mathrm{~mm}$ (111 patients), and into groups with a DRM of $\leq$ $1 \mathrm{~cm}$ (23 patients) and $>1 \mathrm{~cm}$ (105 patients). Table 1 shows the number of survivors and deaths, as also the mortality rate, in these groups by method of operation and in total.

There is a much larger number of patients in the sample with a CRM $>2 \mathrm{~mm}$ and a DRM $>1 \mathrm{~cm}$, there were also far more LAR-s performed than APR-s (evident from Fig. 1).

Survival of patients with DRM $\leq 1 \mathrm{~cm}$ and $>1 \mathrm{~cm}$, and CRM $\leq 2 \mathrm{~mm}$ and $>2 \mathrm{~mm}$ was compared. The number of survivors was noticeably higher than the number of deaths in all groups, regardless of 
operation method. Further analysis with the Mann-Whitney-Wilcoxon test showed statistically significant differences $(p<0.05)$ in survival between the group of patients with a CRM $\leq 2 \mathrm{~mm}$ and $>2 \mathrm{~mm}(\mathrm{p}=$ 0.01396). The survival in the group with CRM $>2 \mathrm{~mm}$ is $20 \%$ higher than those with $\leq 2 \mathrm{~mm}$. If the differences in survival between these groups are compared for individual methods of operation, a statistically significant difference can only be found the for LAR group $(p=0.00635)$, where the survival of patients with CRM $>2 \mathrm{~mm}$ is $26 \%$ higher than those with CRM $\leq 2 \mathrm{~mm}$. A statistically significant difference in survival could not be found $(p=0.1132)$ for patients with different DRM-s. Thus, a better survival for patients with a smaller or larger DRM, cannot be shown within this sample of patients.

The logistic regression model of survival analysis showed that the size of the circumferential margin is an independent predictor $(p=0.0202, A I C=92.181)$ and the size of the DRM is not $(p=0.119, A I C=$ 109.89). The probability of death decreases with the increase of the CRM in a model that uses margin as an independent predictor. Estimated mortality decreased from $30 \%$ to about $10 \%$ for circumferential margin of $2 \mathrm{~mm}$ and larger. Similar results were obtained when the patients operated by the APR method were excluded - suggesting a similar effect in both types of operation. When the circumferential margin and the method of operation were simultaneously included in the model (AIC $=93.886)$, the results show that the size of the circumferential margin is an independent predictor $(p=0.0208)$ and the operative method is not $(p=0.5821)$.

\section{Discussion}

The study showed that a tumor circumferential resection margin of less than $2 \mathrm{~mm}$ were associated with significantly poorer patient survival. This finding was consistent with a great deal of other literature $(6,8)$ and what is accepted in our current clinical practice. The distal resection margin was, not shown to be an independent predictor of survival. Again, consistent with other literature $(12,14,20)$. Thus, a distal magin of less than $1 \mathrm{~cm}$ may be acceptable, if the tumour was completely removed. Unecesserily large distal margins may mean unessecary sacrifice of sphincters in low lying tumours.

Most studies (including the largest of its kind to date (21)), report that a LAR overall produces a higher survival rate than an APR (paradoxically the disease-free survival rates were suggested to be very similar). Though findings were commonly confounded by the fact, that the tumours of the patients were relatively high from the anal verge, and that the subgroups had different PCRT rates $(22,23)$. Most studies on the subject of local failure indicate greater rates with APR. This is most likely because of CRM involvement, which is directly correlated with a more advanced disease stage and larger tumours. These tumours also present lower and are more locally advanced (24), and have a worse response to neoadjuvant therapy (4). Many surgeons still prefer APR in cases of very low tumors with sphincter complex invasion or impaired preoperative state. As such, LAR could be considered an acceptable alternative - especially in patients with a good response to neoadjuvant treatment and adequate margin from the anal canal. 
Because of the aforementioned, the continuation of the intestinal passage is, in clinical practice, desired in nearly every case (25). Despite the benefits offered by the LAR, low anterior resection syndrome (LARS) is a major problem for long term QOL. It usually develops shortly after surgery, then decreases in a few months (the stable state being reached after 2 years). It is more common in patients which have preexisting anorectal dysfunction (especially in women who have given birth - previous trauma to the site of resection (4). Another possible complication is anastomotic leak (may be as high as $10 \%$ ). Current data suggests, that the functional outcome in LAR is superior to APR when no long-term postoperative complications arise - though some studies with objective measurements dispute this $(4,26,27)$. In addition, studies which had a longer follow up suggested, that patients experience a response shift over time, being able to better cope with their condition, the more time passes after surgery. This suggest that planning operations to maximize life expectancy, is linked with an overall higher long term QOL. Thus, smaller DRM, could help the surgeon operate difficult cases, with proper oncological principles, resulting in more favorable long-term survival and functional prognosis. Though caution should still be exercised and so surgeons should not attempt a preferred surgery at any price, but should instead seek to individualize treatment for every case - as well as evaluate for complications both pre and intraoperatively (25). This knowledge could potentially be used to counsel the patient, when conditions for operation, are less than ideal (even when an APR is potentially required). Though, it may be prudent, to view APR as more than a last line treatment in older patients, patients with low life expectancy or major anorectal dysfunction (25).

In our study the APR group ( $D R M>1 \mathrm{~cm}$ ) had a smaller death rate, than the LAR group. This is usually not the case, as studies which report a better prognosis with APR are fewer in number, than those who reporting contrary (23). Despite this the reason for this is most likely neoadjuvant chemotherapy, or PCRT - which may have been used more liberally in cases of patients which are targets for APR, in our sample. The group of patients operated on by the LAR method with a DRM $>1 \mathrm{~cm}$ appears to stand out in the number of deaths. Further analysis showed, however, that it was not an independent variable - it can be assumed that the reason for this is similar $(5,28)$. The difference between mortality in the groups with smaller and larger CRM was also much larger for the LAR method (26\%) than for the APR (6\%). This may, simply be a consequence of neoadjuvant chemotherapy of PCRT (not excluded in this study). An additional factor to consider here, would be the natural coning of the lower bowel which increases chances of CRM involvement in low LAR operations. Apart from this natural coning, there is the issue of coning into the tumor during surgery, due to the higher anastomotic leak rate after complete mesorectal excision (5).

The limitations of the study were small sample size, with resulting small patient subgroups (which varied significantly in size). Despite being designed to minimize differences between its subgroups, they could not be entirely excluded (some may have remained significant). A potential source of bias could be the unequal numbers of APR and LAR operations (the doctors in our institution prefer performing LAR-s) If the patients were controlled for PCRT, the cohort would be so small, as to be beyond any analytical relevance. The data on the effect of PCRT and neoadjuvant therapy on outcomes was inconclusive until recently and was not in the authours scope of view in the time of study design. The FOxTROT trial was 
only published in 2019 and reported a response in the tumour, might make the resection easier, and reduce the risk of incomplete surgical resection. It also reported histological regression in some positive pCRMs. The trial also established, that no increase in perioperative morbidity was seen. This may offer hope for functional outcomes of lower tumours, which are otherwise operable only with APR (29). Some studies have, however reported an increase postoperative anorectal disfunction, when combined with PCRT (30). Another confounding factor, could be the surgical donuts themselves, as a negative stapler donut does not preclude involvement of the distal margin, as it contains only a part of a staple or suture line. This is also true for circumferential margins (there is always a risk of residual malignant cells in the rectal stump) (31).

The results have value in the context of sphincter-sparing resections (DRM-s smaller than $1 \mathrm{~cm}$ may be neccesary in many cases), which may offer significantly better oncological and functional prognosis in select groups of patients. The question of LAR vs. APR in the context of QOL, remains inconclusive, as research results (especially measured with objective questionaires, are scarce and contradictions are common. It can, however be reasoned that a personalised treatment (the precise criteria for this, are still unavailable and may be of interest for future research), less invasive treatment, which does not compromise oncological principles may be optimal for both functional and oncological outcome, especially when the surgeons evaluate for complications during entire treatment.

\section{Conclusion}

In conclusion, the analysis of showed that the CRM was an independent predictor of survival (had a statistically significant effect on survival), while the CRM was not (had no statistically significant effect on patient survival), in our sample. The differences between LAR and APR groups were not statistically significant enough for the operation type to be an independant predictor. As such, a distal resection margin smaller than $1 \mathrm{~cm}$ may not necesserily predict a poor prognosis and may be oncologically acceptable if the tumour was entirely excised. It also reconfirmed the finding, that tumour circumferential resvetion margins of less than $2 \mathrm{~mm}$ are associated with poorer patient survival. This result was noted in many other clinical trials (despite ongoing controvery). This has value in the context for sphincter-sparing resections (DRM-s smaller than $1 \mathrm{~cm}$ may be neccesary in many cases), which may offer significantly better oncological and functional prognosis in select groups of patients.

\section{Abbreviations}

CRC

colorectal cancer; QOL:quality of life; DRM:distal resection margin; CRM:circumferential resection margin; APR:abdominoperineal resection; LAR:low anterior resection; SSR:sphincter sparing resection; TME:total mesenterial excision; pCRM:pathological circumferential resection margin; PCRT:preoperative chemo/radio therapy.

\section{Declarations}


All authors give full consent to publish the manuscript.

Ethics approval and consent to participate: Ethical approval for this study was obtained from the equivalent of the Institutional Review board of our respective institution (University Clinical Centre, Maribor, Slovenia). Informed consent was obtained before any therapeutic procedure took place.

Consent for publication: Not applicable.

Availability of data and materials: The data sets generated and/or analyzed during the current study are not publically available, due to being confidential patient data.

Competing interests: The authors declare that they have no competing interests.

Funding: The authors received no grants, equipment or funding for this study.

Authors' contributions: NA was responsible for study design, interpretation of data. PRR was responsible for data analysis, article drafting and revision.

\section{Acknowledgements}

Not applicable.

\section{References}

1. Bray F, Ferlay J, Soerjomataram I, Siegel RL, Torre LA, Jemal A. Global cancer statistics 2018 : GLOBOCAN estimates of incidence and mortality worldwide for 36 cancers in 185 countries. CA Cancer J Clin [Internet]. 2018 Nov 1;68(6):394-424. Available from: https://doi.org/10.3322/caac.21492

2. Rawla P, Sunkara T, Barsouk A. Epidemiology of colorectal cancer: incidence, mortality, survival, and risk factors. Prz Gastroenterol [Internet]. 2019/01/06. 2019;14(2):89-103. Available from: https://pubmed.ncbi.nlm.nih.gov/31616522

3. Vesna Zadnik et. al. Rak v Sloveniji 2016. Epidemiol Regist raka. 2016;51.

4. Campelo P, Barbosa E. Functional outcome and quality of life following treatment for rectal cancer. J Coloproctology [Internet]. 2016;36(4):251-61. Available from: http://www.sciencedirect.com/science/article/pii/S2237936316300545

5. Miha Lučovnik MO. Resection Margins in Rectal Cancer. Folia Gastrenterol Hepatol. 2008;6(1).

6. Liu Q, Luo D, Cai S, Li Q, Li X. Circumferential resection margin as a prognostic factor after rectal cancer surgery: A large population-based retrospective study. Cancer Med [Internet]. 2018/07/10. 2018 Aug;7(8):3673-81. Available from: https://pubmed.ncbi.nlm.nih.gov/29992773

7. Hellinger MD, Santiago CA. Reoperation for recurrent colorectal cancer. Clin Colon Rectal Surg [Internet]. 2006 Nov;19(4):228-36. Available from: https://pubmed.ncbi.nlm.nih.gov/20011326 
8. Carlsen E, Schlichting E, Guldvog I, Johnson E, Heald RJ. Effect of the introduction of total mesorectal excision for the treatment of rectal cancer. Br J Surg. 1998 Apr;85(4):526-9.

9. Lirici MM, Hüscher CGS. Techniques and technology evolution of rectal cancer surgery: a history of more than a hundred years. Minim Invasive Ther Allied Technol [Internet]. 2016 Sep 2;25(5):226-33. Available from: https://doi.org/10.1080/13645706.2016.1198381

10. Delibegovic S. Introduction to Total Mesorectal Excision. Med Arch (Sarajevo, Bosnia Herzegovina) [Internet]. 2017 Dec;71(6):434-8. Available from: https://pubmed.ncbi.nIm.nih.gov/29416206

11. Ahuja V. 14 - Open Surgical Techniques in Colorectal Cancer. In: Gearhart SL, Ahuja NBT-ED and T of CSCC, editors. 1. Saint Louis: W.B. Saunders; 2011. p. 145-65. Available from: http://www.sciencedirect.com/science/article/pii/B9781416046868500191

12. Grosek J, Velenik V, Edhemovic I, Omejc M. The Influence of the Distal Resection Margin Length on Local Recurrence and long- term Survival in Patients with Rectal Cancer after Chemoradiotherapy and Sphincter- Preserving Rectal Resection. Radiol Oncol [Internet]. 2016 May 24;51(2):169-77. Available from: https://pubmed.ncbi.nlm.nih.gov/28740452

13. Heald RJ, Ryall RDH. RECURRENCE AND SURVIVAL AFTER TOTAL MESORECTAL EXCISION FOR RECTAL CANCER. Lancet [Internet]. 1986;327(8496):1479-82. Available from: http://www.sciencedirect.com/science/article/pii/S0140673686915102

14. Park IJ, Kim JC. Adequate Length of the Distal Resection Margin in Rectal Cancer: From the Oncological Point of View. J Gastrointest Surg [Internet]. 2010;14(8):1331-7. Available from: https://doi.org/10.1007/s11605-010-1165-3

15. Hong KS, Moon N, Chung SS, Lee R-A, Kim KH. Oncologic outcomes in rectal cancer with close distal resection margins: a retrospective analysis. Ann Surg Treat Res [Internet]. 2015/06/11. 2015 Jul;89(1):23-9. Available from: https://pubmed.ncbi.nlm.nih.gov/26131441

16. Rentsch M, Schiergens T, Khandoga A, Werner J. Surgery for Colorectal Cancer - Trends, Developments, and Future Perspectives. Visc Med [Internet]. 2016/06/13. 2016 Jun;32(3):184-91. Available from: https://pubmed.ncbi.nlm.nih.gov/27493946

17. Nishikawa T, Ishihara S, Emoto S, Kaneko M, Murono K, Sasaki K, et al. Multivisceral resections for locally advanced colorectal cancer after preoperative treatment. Mol Clin Oncol [Internet]. 2018/01/19. 2018 Mar;8(3):493-8. Available from: https://pubmed.ncbi.nlm.nih.gov/29564129

18. Pędziwiatr M, Mizera M, Witowski J, Major P, Torbicz G, Gajewska N, et al. Primary tumor resection in stage IV unresectable colorectal cancer: what has changed? Med Oncol [Internet]. 2017 Oct 30;34(12):188. Available from: https://pubmed.ncbi.nlm.nih.gov/29086041

19. Sudo M, Furuya S, Shimizu H, Nakata Y, lino H, Shiraishi K, et al. Long-term outcomes after surgical resection in patients with stage IV colorectal cancer: a retrospective study of 129 patients at a single institution. World J Surg Oncol [Internet]. 2019;17(1):56. Available from:

https://doi.org/10.1186/s12957-019-1599-3

20. Bujko K, Rutkowski A, Chang GJ, Michalski W, Chmielik E, Kusnierz J. Is the 1-cm rule of distal bowel resection margin in rectal cancer based on clinical evidence? A systematic review. Ann Surg Oncol 
[Internet]. 2011/08/31. 2012 Mar;19(3):801-8. Available from:

https://pubmed.ncbi.nlm.nih.gov/21879269

21. Saito N, Sugito M, Ito M, Kobayashi A, Nishizawa $Y$, Yoneyama $Y$, et al. Oncologic outcome of intersphincteric resection for very low rectal cancer. World J Surg. 2009 Aug;33(8):1750-6.

22. Yeom S-S, Park IJ, Jung SW, Oh SH, Lee JL, Yoon YS, et al. Outcomes of patients with abdominoperineal resection (APR) and low anterior resection (LAR) who had very low rectal cancer. Medicine (Baltimore) [Internet]. 2017 Oct;96(43):e8249-e8249. Available from: https://pubmed.ncbi.nlm.nih.gov/29068989

23. Wang X-T, Li D-G, Li L, Kong F-B, Pang L-M, Mai W. Meta-analysis of oncological outcome after abdominoperineal resection or low anterior resection for lower rectal cancer. Pathol Oncol Res [Internet]. 2014/11/28. 2015 Jan;21(1):19-27. Available from:

https://pubmed.ncbi.nlm.nih.gov/25430561

24. Omidvari S, Hamedi SH, Mohammadianpanah M, Razzaghi S, Mosalaei A, Ahmadloo N, et al. Comparison of abdominoperineal resection and low anterior resection in lower and middle rectal cancer. J Egypt Natl Canc Inst [Internet]. 2013;25(3):151-60. Available from: http://www.sciencedirect.com/science/article/pii/S1110036213000630

25. Turan M. Comparison of Quality of Life Scores in Patients Who Underwent Low Anterior Resection and Abdominoperineal Resection for Tumors of The Rectum. Biomed J Sci Tech Res. 2019 Aug $26 ; 20$.

26. Campos-Lobato LF de, Alves-Ferreira PC, Lavery IC, Kiran RP. Abdominoperineal resection does not decrease quality of life in patients with low rectal cancer. Clinics [Internet]. 2011;66:1035-40. Available from: http://www.scielo.br/scielo.php?script=sci_arttext\&pid=S180759322011000600019\&nrm=iso

27. Wani RA, Bhat I-U-A, Parray FQ, Chowdri NA. Quality of Life After "Total Mesorectal Excision (TME)" for Rectal Carcinoma: a Study from a Tertiary Care Hospital in Northern India. Indian J Surg Oncol [Internet]. 2017/08/25. 2017 Dec;8(4):499-505. Available from:

https://pubmed.ncbi.nlm.nih.gov/29203980

28. Beck DE. Surgical management of colon and rectal cancer. Ochsner J [Internet]. 2002;4(3):156-62. Available from: https://pubmed.ncbi.nlm.nih.gov/22822339

29. Seymour MT, Morton D. FOxTROT: an international randomised controlled trial in 1052 patients (pts) evaluating neoadjuvant chemotherapy (NAC) for colon cancer. J Clin Oncol [Internet]. 2019 May 20;37(15_suppl):3504. Available from: https://doi.org/10.1200/JC0.2019.37.15_suppl.3504

30. Fernández-Martínez D, Rodríguez-Infante A, Otero-Díez JL, Baldonedo-Cernuda RF, Mosteiro-Díaz MP, García-Flórez LJ. Is my life going to change?-a review of quality of life after rectal resection. J Gastrointest Oncol [Internet]. 2020 Feb;11(1):91-101. Available from: https://pubmed.ncbi.nlm.nih.gov/32175110

31. Chowdri NA. Donuts and Safe Margins in Rectal Cancer Resection. Clin Surg. 2018;3. 


\section{Tables}

Due to technical limitations, table 1 is only available as a download in the Supplemental Files section.

\section{Figures}

\section{Figure 1.}

(a)

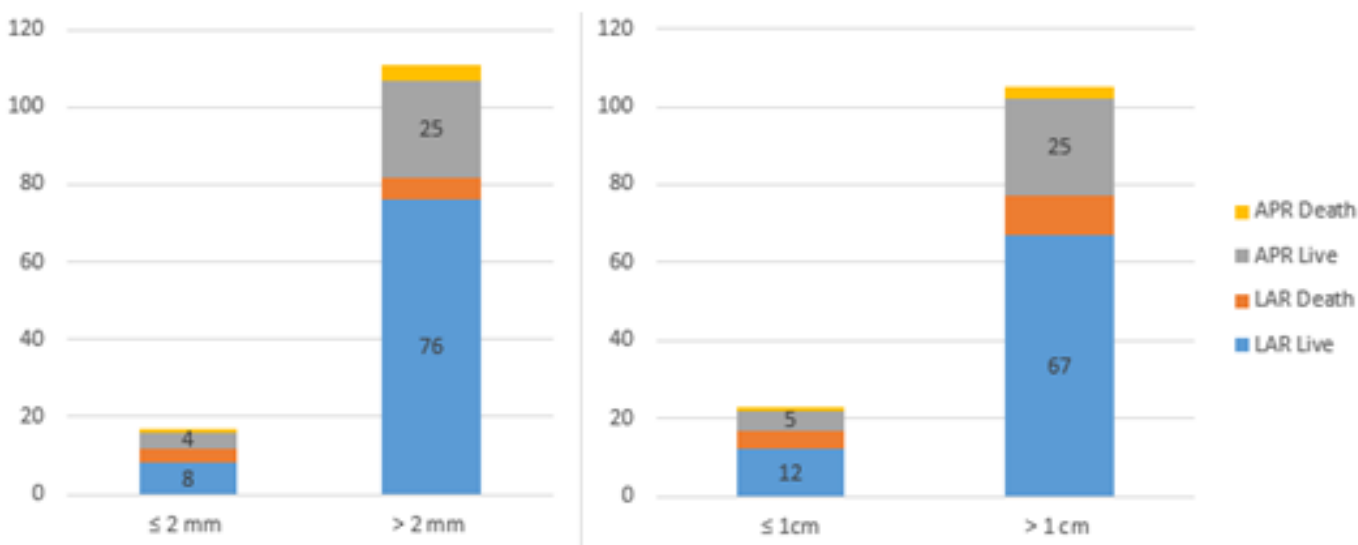

(b)

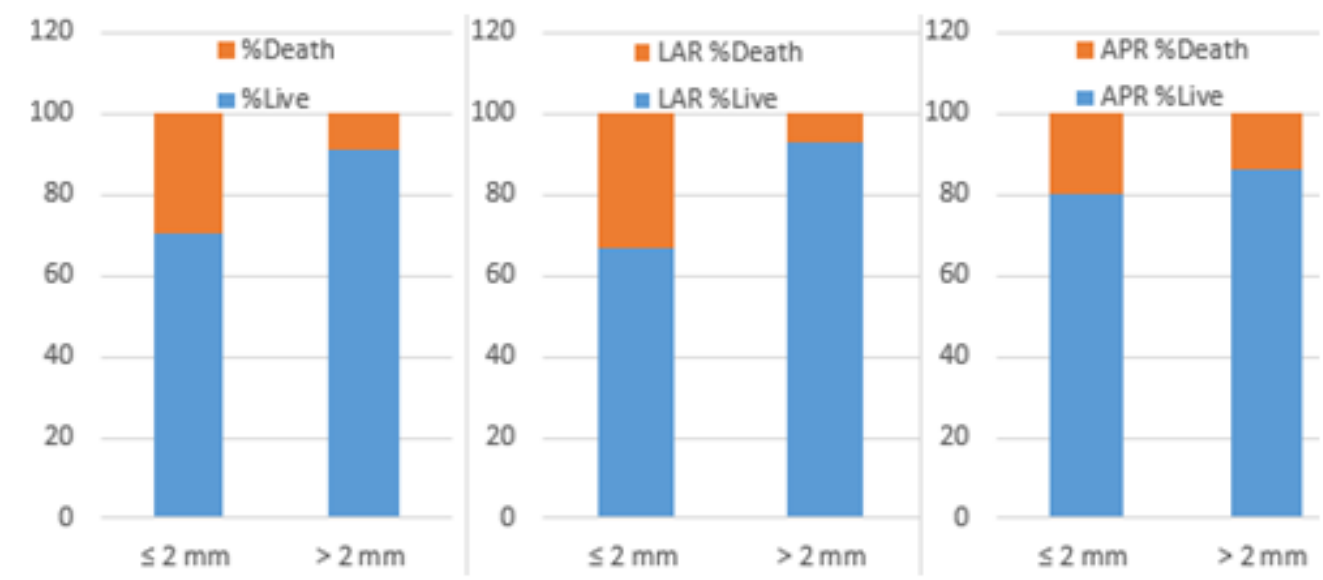

(c)
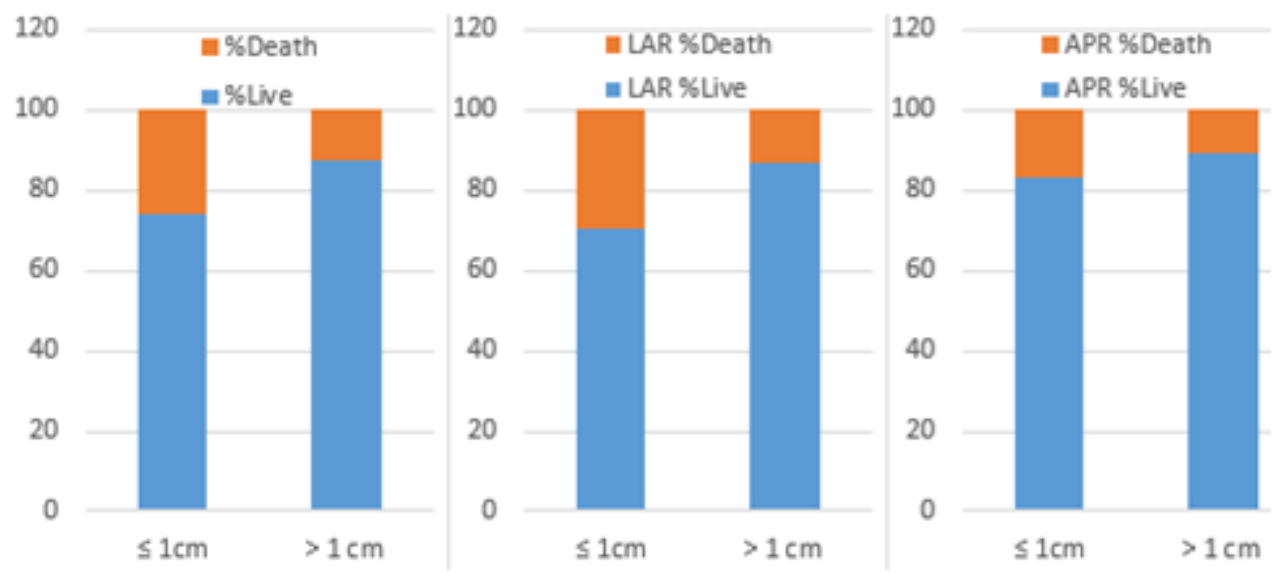

Figure 1 
Number of lives and deaths (a) and their shares for circumferential (b) and distal (c) margins for LAR and APR methods

\section{Supplementary Files}

This is a list of supplementary files associated with this preprint. Click to download.

- BMCsurgeryTable1.PNG 